MATHEMATICS OF COMPUTATION

Volume 73, Number 246, Pages 731-751

S $0025-5718(03) 01594-1$

Article electronically published on July 29, 2003

\title{
APPROXIMATING THE JUMP DISCONTINUITIES OF A FUNCTION BY ITS FOURIER-JACOBI COEFFICIENTS
}

\author{
GEORGE KVERNADZE
}

\begin{abstract}
In the present paper we generalize Eckhoff's method, i.e., the method for approximating the locations of discontinuities and the associated jumps of a piecewise smooth function by means of its Fourier-Chebyshev coefficients.

A new method enables us to approximate the locations of discontinuities and the associated jumps of a discontinuous function, which belongs to a restricted class of the piecewise smooth functions, by means of its Fourier-Jacobi coefficients for arbitrary indices. Approximations to the locations of discontinuities and the associated jumps are found as solutions of algebraic equations. It is shown as well that the locations of discontinuities and the associated jumps are recovered exactly for piecewise constant functions with a finite number of discontinuities.

In addition, we study the accuracy of the approximations and present some numerical examples.
\end{abstract}

\section{INTRODUCTION}

An important assumption for a number of spectral methods designed for the reconstruction of a piecewise smooth function is the accurate knowledge of the function's discontinuity locations and the associated jumps. This key data should be extracted from spectral modes of a given function.

A number of authors (see Banerjee and Geer [1], 8], Bauer [2, Cai et al. [3], Eckhoff [4, [5], [6, Gelb and Tadmor [9], [10, Kvernadze et al. [13, [14], 15], 16], and Mhaskar and Prestin [20]) studied the problem of approximating the singularity locations and the associated jumps of a piecewise smooth function given a finite number of its ordinary Fourier coefficients.

Eckhoff [4] introduced the first explicit method to recover the discontinuities of a piecewise smooth function by means of its Fourier coefficients with respect to an orthonormal system of algebraic polynomials. He developed a method to approximate the locations of discontinuities and the associated jumps of a piecewise smooth function by means of its Fourier-Chebyshev coefficients. If a function has a finite number, $M$, of jump discontinuities, then approximations to the locations of discontinuities are found as solutions of certain $M$ th degree algebraic equation.

Mhaskar and Prestin [18, [19] proposed a class of algebraic polynomial frames that can be used to detect discontinuities in derivatives of all orders of a function.

Received by the editor November 30, 2001 and, in revised form, November 21, 2002.

2000 Mathematics Subject Classification. Primary 65D99, 65T99, 42C10.

Key words and phrases. Approximating the jump discontinuities, Fourier-Jacobi coefficients. 
Recently Gelb and Tadmor [9], [10] used a different approach by introducing socalled "concentration kernels" $K_{\epsilon}(\cdot)$, depending on the small scale $\epsilon$. These satisfy the condition $K_{\epsilon} * f(x)=f(x+)-f(x-)+O(\epsilon)$, thus recovering both the location and the amplitude of all singularities. In particular, the authors have considered concentration kernels with respect to the Gegenbauer system of orthonormal polynomials with nonpositive indices. A special case corresponding to the polynomial concentration kernel with respect to the Fourier-Jacobi series was studied by the author earlier in $[13$.

In the present paper we investigate the problem of approximating the jump discontinuity locations of a bounded function given its finite number of FourierJacobi coefficients. As a result, a new method is developed which enables us to approximate the locations of discontinuities and the associated jumps of a function of the $\tilde{C}^{r}[-1,1]$ class (see Definition 2.1) by solving appropriate algebraic equations. Theorem 3.1 shows that the locations of discontinuities and the associated jumps can be recovered exactly for piecewise constant functions with a finite number of discontinuities. In Theorems 3.2 and 3.3 it is proved, and subsequently numerically confirmed, that for functions of the $\tilde{C}^{3}[-1,1]$ class the locations of discontinuities are approximated to within $O\left(1 / n^{2}\right)$ and the associated jumps to within $O(1 / n)$.

\section{Preliminaries}

Throughout this paper we use the following general notations: $N, Z_{+}$, and $R$ are the sets of positive integers, nonnegative integers, and real numbers, respectively. By $\mathbf{b}=\left(b_{1}, b_{2}, \ldots, b_{M}\right) \in R^{M}$ we denote a column vector, where $b_{m} \in R, m=$ $1,2, \ldots, M$, and $\|\mathbf{b}\|=\max _{1 \leq m \leq M}\left|b_{m}\right|$ is the $\ell_{\infty}$ norm of the vector $\mathbf{b}$. If $\mathbf{A}$ is a $M \times M$ matrix, by $\|\mathbf{A}\|=\sup _{\|\mathbf{b}\|=1}\|\mathbf{A b}\| /\|\mathbf{b}\|$ we denote its natural (induced) $\ell_{\infty}$ norm.

By $C^{-1}[a, b]$ we denote the space of functions on $[a, b]$ that may have only a finite number of jump discontinuities and are normalized by the condition $f(x)=$ $(f(x+)+f(x-)) / 2$ (here and elsewhere $f(x+)$ and $f(x-)$ denote the right-hand and left-hand side limits of the function $f$ at a point $x)$.

By $[f](x) \equiv f(x+)-f(x-)$ we denote the jump of the function $f \in C^{-1}[-1,1]$ at the point $x$. By $M \equiv M(f)$ we denote the number of discontinuities of the function $f \in C^{-1}[-1,1]$ and by $x_{m} \equiv x_{m}(f), m=1, \ldots, M$, we denote the points of discontinuity of the function $f \in C^{-1}[-1,1]$ arranged in increasing order.

By $C^{r}[a, b], r \in Z_{+}$, we denote the space of $r$-times continuously differentiable functions on $[a, b]$.

Definition 2.1. We say that a function $f$ belongs to $\tilde{C}^{r}[a, b]$ if there exist points $a=x_{0}<x_{1}<\ldots<x_{M}<x_{M+1}=b, M<\infty$, such that the function $f$ is $r$-times continuously differentiable over each interval $\left(x_{m}, x_{m+1}\right), m=0,1, \ldots, M$, one-sided limits of the function and its derivatives up to $r$ th derivative at the points $x_{m}$ exist and are finite, and $[f]\left(x_{m}\right) \equiv\left[f^{(0)}\right]\left(x_{m}\right) \neq 0, m=1,2, \ldots, M$.

By $K$ we denote constants, possibly depending on some fixed parameters, and in general distinct in different formulas. Sometimes the important arguments of $K$ will be written explicitly in the expressions for it. For quantities $A_{n}$ and $B_{n}$, possibly depending on some other variables as well, we write $A_{n}=o\left(B_{n}\right)$ or $A_{n}=O\left(B_{n}\right)$, if $\lim _{n \rightarrow \infty} A_{n} / B_{n}=0$ or $\sup _{n \in N}\left|A_{n} / B_{n}\right|<\infty$, respectively. For quantities $A$ and 
$B$, depending on some variables, we write $A \sim B$ if the ratio $A / B$ is between two positive constants, independent of the variables.

We say that $\rho^{(\alpha, \beta)}$ is a Jacobi weight if $\rho^{(\alpha, \beta)}(x)=(1-x)^{\alpha}(1+x)^{\beta}, \alpha>-1$ and $\beta>-1$. If $\rho^{(\alpha, \beta)}$ is a Jacobi weight, then by $\sigma\left(\rho^{(\alpha, \beta)}\right)=\left(P_{n}^{(\alpha, \beta)}(x)\right)_{n=0}^{\infty}$ we denote the corresponding system of orthogonal polynomials $P_{n}^{(\alpha, \beta)}(x)=\gamma_{n}(\alpha, \beta) x^{n}+$ lower degree terms, $\gamma_{n}(\alpha, \beta)>0$, normalized by the condition $P_{n}^{(\alpha, \beta)}(1)=\left(\begin{array}{c}n+\alpha \\ n\end{array}\right), n \in N$; i.e.,

$$
\int_{-1}^{1} P_{n}^{(\alpha, \beta)}(x) P_{m}^{(\alpha, \beta)}(x) \rho^{(\alpha, \beta)}(x) d x=0, \quad n \neq m .
$$

The system $\sigma\left(\rho^{(\alpha, \beta)}\right)$ is defined uniquely and is called the Jacobi system of orthogonal polynomials. Some important special cases of the Jacobi system are the Chebyshev $(\alpha=\beta=-1 / 2)$, Legendre $(\alpha=\beta=0)$, and Gegenbauer $(\alpha=\beta)$ systems.

If $f \rho^{(\alpha, \beta)}$ is integrable on $[-1,1]$, then the function $f$ has a Fourier series with respect to the system $\sigma\left(\rho^{(\alpha, \beta)}\right)$, and by $S_{n}^{(\alpha, \beta)}(f, x)$ we denote the $n$th partial sum of the Fourier series of $f$ with respect to the system $\sigma\left(\rho^{(\alpha, \beta)}\right)$; i.e.,

$$
S_{n}^{(\alpha, \beta)}(f, x)=\sum_{k=0}^{n} \mu_{k}^{(\alpha, \beta)} a_{k}^{(\alpha, \beta)}(f) P_{k}^{(\alpha, \beta)}(x),
$$

where

$$
a_{k}^{(\alpha, \beta)}(f)=\int_{-1}^{1} f(t) P_{k}^{(\alpha, \beta)}(t) \rho^{(\alpha, \beta)}(t) d t
$$

is the $k$ th Fourier coefficient of the function $f$ and $\mu_{k}^{(\alpha, \beta)} \sim k$.

To avoid unnecessary complication of notation, we sometimes omit dependence on parameters $\alpha>-1$ and $\beta>-1$, as they are arbitrary, but fixed.

By virtue of the Christoffel-Darboux formula

$P_{n+1}^{(\alpha, \beta)}(x) P_{n}^{(\alpha, \beta)}(t)-P_{n}^{(\alpha, \beta)}(x) P_{n+1}^{(\alpha, \beta)}(t)=(x-t) h_{n}^{(\alpha, \beta)} \sum_{k=0}^{n} \mu_{k}^{(\alpha, \beta)} P_{k}^{(\alpha, \beta)}(x) P_{k}^{(\alpha, \beta)}(t)$

for $n \in N$, where $h_{n}^{(\alpha, \beta)} \sim 1 / n$.

If $x_{k, n}^{(\alpha, \beta)}, k=1,2, \ldots, n$, are the zeros of the polynomial $P_{n}^{(\alpha, \beta)}(x)$, then

$$
x_{k, n+1}^{(\alpha, \beta)}<x_{k, n}^{(\alpha, \beta)}<x_{k+1, n+1}^{(\alpha, \beta)} .
$$

The asymptotic formula

$$
P_{n}^{(\alpha, \beta)}(\cos \tau)=K(\alpha, \beta, \tau)\left[\cos (\tilde{n} \tau+\tilde{\gamma})+O(1)(n \sin \tau)^{-1}\right],
$$

holds as $n \rightarrow \infty$, where

$$
\begin{aligned}
K(\alpha, \beta, \tau) & =2^{-(\alpha+\beta) / 2} \pi^{-1 / 2} \sin ^{-\alpha-1 / 2}(\tau / 2) \cos ^{-\beta-1 / 2}(\tau / 2), \\
\tilde{n} & =n+(\alpha+\beta+1) / 2, \quad \tilde{\gamma}=-(2 \alpha+1) \pi / 4,
\end{aligned}
$$

and

$$
K / n \leq \tau \leq \pi-K / n .
$$

The following is a generalization of Rodrigues' formula:

$$
\rho^{(\alpha, \beta)}(x) P_{k}^{(\alpha, \beta)}(x)=\frac{(-1)^{i}(k-i) !}{2^{i} k !} \frac{d^{i}}{d x^{i}}\left(\rho^{(\alpha+i, \beta+i)}(x) P_{k-i}^{(\alpha+i, \beta+i)}(x)\right),
$$

where $k \geq i$. 
This is the recurrence formula for the Jacobi polynomials

$$
A_{k}^{(\alpha, \beta)} P_{k+1}^{(\alpha, \beta)}(x)+\left(x+B_{k}^{(\alpha, \beta)}\right) P_{k}^{(\alpha, \beta)}(x)+C_{k}^{(\alpha, \beta)} P_{k-1}^{(\alpha, \beta)}(x)=0,
$$

where

$$
\begin{aligned}
& A_{k}^{(\alpha, \beta)}=-\frac{2(k+1)(k+\alpha+\beta+1)}{(2 k+\alpha+\beta+1)(2 k+\alpha+\beta+2)}=O(1), \\
& B_{k}^{(\alpha, \beta)}=\frac{\alpha^{2}-\beta^{2}}{(2 k+\alpha+\beta+2)(2 k+\alpha+\beta)}=O\left(\frac{1}{k^{2}}\right), \\
& C_{k}^{(\alpha, \beta)}=-\frac{2(k+\alpha)(k+\beta)}{(2 k+\alpha+\beta+1)(2 k+\alpha+\beta)}=O(1)
\end{aligned}
$$

for $k \geq 2$ and $P_{0}^{(\alpha, \beta)}(x)=1$ and $P_{1}^{(\alpha, \beta)}(x)=(\alpha+\beta+2) x / 2+(\alpha-\beta) / 2$.

Let us mention an obvious consequence of (2.6):

$$
\begin{aligned}
& A_{k-i}^{(\alpha+i, \beta+i)}=A_{k}^{(\alpha, \beta)}+\frac{2 i(\alpha+\beta+i)}{(2 k+\alpha+\beta+1)(2 k+\alpha+\beta+2)}=A_{k}^{(\alpha, \beta)}+O\left(\frac{1}{k^{2}}\right), \\
& B_{k-i}^{(\alpha+i, \beta+i)}=B_{k}^{(\alpha, \beta)}+\frac{2 i(\alpha-\beta)}{(2 k+\alpha+\beta)(2 k+\alpha+\beta+2)}=B_{k}^{(\alpha, \beta)}+O\left(\frac{1}{k^{2}}\right), \\
& C_{k-i}^{(\alpha+i, \beta+i)}=C_{k}^{(\alpha, \beta)}
\end{aligned}
$$

for fixed $i \in N$ and $k \geq i+2$.

$$
\left|P_{k-1}^{(\alpha, \beta)}(x)\right|<K(\alpha, \beta) k^{-1 / 2}\left((1-x)^{1 / 2}+\frac{1}{k}\right)^{-\alpha-1 / 2}\left((1+x)^{1 / 2}+\frac{1}{k}\right)^{-\beta-1 / 2}
$$

holds for $x \in[-1,1]$ and $k \in N$. (Regarding (2.1)-(2.5) and (2.8), see [22. pp. 71, $46,197,97,71$, and 169.])

The following is a function which has a jump discontinuity of order $i \in Z_{+}$, i.e., $\left[f^{(s)}\right](x)=0, s=0,1, \ldots, i-1$, and $\left[f^{(i)}\right](x) \neq 0$, with magnitude 1 at the point $x \in(-1,1)$ and which is smooth everywhere else:

$$
H_{i}(x, t) \equiv \begin{cases}0, & \text { if }-1 \leq t<x, \\ \frac{(t-x)^{i}}{i !}, & \text { if } x<t \leq 1\end{cases}
$$

Therefore, a given function $f \in \tilde{C}^{r}[-1,1]$ can be expressed as follows:

$$
f=\sum_{i=0}^{r} \sum_{m=1}^{M}\left[f^{(i)}\right]\left(x_{m}\right) H_{i}\left(x_{m}, \cdot\right)+f_{c},
$$

where $f_{c}$ is $r$-times continuously differentiable on $[-1,1]$.

Obviously, the smoother $f_{c}$ is, the more rapidly $a_{k}^{(\alpha, \beta)}\left(f_{c}\right)$ converges to zero. Hence,

$$
a_{k}^{(\alpha, \beta)}(f) \approx \sum_{i=0}^{r} \sum_{m=1}^{M}\left[f^{(i)}\right]\left(x_{m}\right) a_{k}^{(\alpha, \beta)}\left(H_{i}\left(x_{m}, \cdot\right)\right)
$$

and it is plausible to recover the information about the locations of discontinuities and the associated jumps of a given function from its Fourier-Jacobi coefficients. 
It is easy to check that (see (2.4), (2.8), and (2.9))

$$
\begin{aligned}
& a_{k}^{(\alpha, \beta)}\left(H_{i}(x, \cdot)\right)=\frac{(k-i-1) !}{2^{i+1} k !} \rho^{(\alpha+i+1, \beta+i+1)}(x) P_{k-i-1}^{(\alpha+i+1, \beta+i+1)}(x) \\
& \quad= \begin{cases}O\left(k^{-i-3 / 2}\right) \rho^{(\alpha / 2+i / 2+1 / 4, \beta / 2+i / 2+1 / 4)}(x), & \text { if } x \in\left[-1+\frac{1}{k^{2}}, 1-\frac{1}{k^{2}}\right], \\
O\left(k^{\max (\alpha, \beta)}\right) \rho^{(\alpha+i+1, \beta+i+1)}(x), & \text { if } x \in[-1,1] \backslash\left[-1+\frac{1}{k^{2}}, 1-\frac{1}{k^{2}}\right],\end{cases}
\end{aligned}
$$

holds for $k>i, i \in Z_{+}$.

For a given function $f$, the polynomial of $i$ variables

$$
a_{k}^{(i)}\left(t_{1}, t_{2}, \ldots, t_{i}\right) \equiv a_{k}^{(i)}\left(f, t_{1}, t_{2}, \ldots, t_{i}\right), \quad t_{i} \in R, i=1,2, \ldots, M,
$$

is defined as

$$
a_{k}^{(0)} \equiv a_{k}^{(0)}(f) \equiv 2(k+1) a_{k+1}^{(\alpha, \beta)}(f)
$$

for $k \in Z_{+}$and

$$
\begin{aligned}
a_{k}^{(i)}\left(t_{1}, t_{2}, \ldots, t_{i}\right) \equiv & A_{k}^{(\alpha+1, \beta+1)} a_{k+1}^{(i-1)}\left(t_{1}, t_{2}, \ldots, t_{i-1}\right) \\
& +\left(t_{i}+B_{k}^{(\alpha+1, \beta+1)}\right) a_{k}^{(i-1)}\left(t_{1}, t_{2}, \ldots, t_{i-1}\right) \\
& +C_{k}^{(\alpha+1, \beta+1)} a_{k-1}^{(i-1)}\left(t_{1}, t_{2}, \ldots, t_{i-1}\right)
\end{aligned}
$$

for $i \in N$ and $k \geq i$, where $a_{k}^{(1)}\left(t_{1}\right) \equiv A_{k}^{(\alpha+1, \beta+1)} a_{k+1}^{(0)}+\left(t_{1}+B_{k}^{(\alpha+1, \beta+1)}\right) a_{k}^{(0)}+$ $C_{k}^{(\alpha+1, \beta+1)} a_{k-1}^{(0)}$.

A particular value of the polynomial $a_{k}^{(i)}\left(t_{1}, t_{2}, \ldots, t_{i}\right)$, namely

$$
a_{k}^{(i)} \equiv a_{k}^{(i)}(0,0, \ldots, 0)
$$

will be called a higher order Fourier-Jacobi coefficient of the function $f$.

For a function $f \in C^{-1}[-1,1]$ with $M$ jump discontinuities we introduce the $M \times M$ matrix

$$
\mathbf{A}_{M}^{(n)} \equiv \mathbf{A}_{M}^{(n)}(f) \equiv\left(a_{n+j}^{(i)}\right)_{i, j=0}^{M-1}
$$

If $\mathbf{A}_{M}^{(n)}$ is nonsingular for some $n \geq M$, then by $x_{m}(n) \equiv x_{m}(f, n), m=$ $1,2, \ldots, M$, we denote the solutions of the polynomial equation

$$
Q_{M}^{(n)}(x) \equiv x^{M}+\sum_{i=1}^{M}(-1)^{i} q_{M-i}^{(M)}(n) x^{M-i}=0
$$

where $q_{i}^{(M)}(n), i=0,1, \ldots, M-1$, are determined by the linear equations

$$
\sum_{i=0}^{M-1} a_{n+j}^{(i)} q_{i}^{(M)}(n)+a_{n+j}^{(M)}=0 \quad(j=0,1, \ldots, M-1) .
$$

We also consider the matrices

$$
\mathbf{P}_{M}^{(n)} \equiv \mathbf{P}_{M}^{(n)}\left(t_{1}, t_{2}, \ldots, t_{M}\right) \equiv\left(\mathbf{p}_{n}^{(M)}, \mathbf{p}_{n+1}^{(M)}, \ldots, \mathbf{p}_{n+M-1}^{(M)}\right) \equiv\left(P_{n+j}^{(\alpha+1, \beta+1)}\left(t_{i+1}\right)\right)_{i, j=0}^{M-1}
$$

for $n \geq M$ and $t_{m} \in R, m=1,2, \ldots, M$, where $\mathbf{p}_{s}^{(M)} \equiv\left(P_{s}^{(\alpha+1, \beta+1)}\left(t_{1}\right), P_{s}^{(\alpha+1, \beta+1)}\left(t_{2}\right), \ldots, P_{s}^{(\alpha+1, \beta+1)}\left(t_{M}\right)\right) \in R^{M}$ is the $s$ th column vector of the matrix $\mathbf{P}_{M}^{(n)}$. 
If $x_{m}(n), m=1,2, \ldots, M$, represent solutions of the equation (2.15) for some $n \geq M$ and $P_{k}^{(\alpha+1, \beta+1)}\left(x_{m}(n)\right) \neq 0$ for some $k \sim n$, then $f_{m}(n), m=1,2, \ldots, M$, is determined by the equation

$$
\begin{aligned}
f_{m}(n) \rho^{(\alpha+1, \beta+1)} & \left(x_{m}(n)\right) P_{k}^{(\alpha+1, \beta+1)}\left(x_{m}(n)\right) \prod_{s=1 ; s \neq m}^{M}\left(x_{s}(n)-x_{m}(n)\right) \\
= & a_{k}^{(M-1)}\left(x_{1}(n), \ldots, x_{m-1}(n), x_{m+1}(n), \ldots, x_{M}(n)\right) .
\end{aligned}
$$

Let us give some explanations to the notions introduced above.

First, via recurrence formulas (2.12) and (2.13), we have constructed the polynomials $a_{k}^{(i)}\left(t_{1}, t_{2}, \ldots, t_{i}\right), k \geq i$, utilizing given Fourier-Jacobi coefficients of the function $f$. Next, we considered the system of linear equations (2.16) using higher order Fourier-Jacobi coefficients of a given function. Under assumption that the linear system of equations is consistent, i.e., $\operatorname{det} \mathbf{A}_{M}^{(n)} \neq 0$ (see (2.14) ), we used its solution to build the polynomial $Q_{M}^{(n)}$; see (2.15). It will be shown that the solutions $x_{m}(n), m=1,2, \ldots M$, of the polynomial equation 2.15) represent approximations to the locations of discontinuities and the solutions of linear equations (2.18) represent approximations to the magnitudes of the jumps of the given function $f \in \tilde{C}^{3}[-1,1]$.

In what follows, in Lemma 2.2 we will derive a key identity which relates the polynomials $a_{k}^{(i)}\left(f, t_{1}, t_{2}, \ldots, t_{i}\right)$, associated to a piecewise constant function $f$ with a finite number, $M$, of discontinuities, to the locations of discontinuities of the function and the corresponding jumps; see (2.19). As a corollary we will learn that the zeros of the polynomial $a_{k}^{(M)}\left(t_{1}, t_{2}, \ldots, t_{M}\right)$ represent the locations of discontinuities $x_{m}, m=1,2, \ldots, M$, of the given piecewise constant function $f$, i.e., $a_{k}^{(M)}\left(x_{1}, x_{2}, \ldots, x_{M}\right)=0 ;$ see (2.23).

Next, in Lemma 2.3, we will show that there is the close relation (2.27) between higher order Fourier-Jacobi coefficients of a function $f \in C^{-1}[-1,1]$, the associated polynomials $a_{k}^{(M)}\left(t_{1}, t_{2}, \ldots, t_{M}\right)$, and the coefficients of the polynomial (2.15).

Now, we formulate and give formal proofs for Lemmas 2.2 and 2.3.

Lemma 2.2. Let $f$ be a piecewise constant function defined on $[-1,1]$ with a finite number, $M$, of discontinuities at the points $x_{m}, m=1,2, \ldots, M$. Then

$$
a_{k}^{(i)}\left(f, t_{1}, t_{2}, \ldots, t_{i}\right)=\sum_{m=1}^{M}[f]\left(x_{m}\right) \rho^{(\alpha+1, \beta+1)}\left(x_{m}\right) P_{k}^{(\alpha+1, \beta+1)}\left(x_{m}\right) \prod_{s=1}^{i}\left(t_{s}-x_{m}\right),
$$

where $k \geq i$.

Proof. Since the function $f$ is piecewise constant, it may be represented as

$$
f=\sum_{m=1}^{M}[f]\left(x_{m}\right) H_{0}\left(x_{m}, \cdot\right)+f(-1+) .
$$

Then by 2.11), 2.12), and (2.20) we get

$$
a_{k}^{(0)}=\sum_{m=1}^{M}[f]\left(x_{m}\right) \rho\left(x_{m}\right) P_{k}\left(x_{m}\right)
$$

for $k \in N$, where $\rho(x) \equiv \rho^{(\alpha+1, \beta+1)}(x)$ and $P_{k}(x) \equiv P_{k}^{(\alpha+1, \beta+1)}(x)$. 
By virtue of (2.5), (2.13), and (2.21) we have

$$
\begin{aligned}
a_{k}^{(1)}\left(t_{1}\right) & =A_{k} a_{k+1}^{(0)}+\left(t_{1}+B_{k}\right) a_{k}^{(0)}+C_{k} a_{k-1}^{(0)} \\
& =\sum_{m=1}^{M}[f]\left(x_{m}\right) \rho\left(x_{m}\right)\left[A_{k} P_{k+1}\left(x_{m}\right)+\left(t_{1}+B_{k}\right) P_{k}\left(x_{m}\right)+C_{k} P_{k-1}\left(x_{m}\right)\right] \\
& =\sum_{m=1}^{M}[f]\left(x_{m}\right) \rho\left(x_{m}\right) P_{k}\left(x_{m}\right)\left(t_{1}-x_{m}\right)
\end{aligned}
$$

for $k \geq 1$, where $A_{k} \equiv A_{k}^{(\alpha+1, \beta+1)}, B_{k} \equiv B_{k}^{(\alpha+1, \beta+1)}$, and $C_{k} \equiv C_{k}^{(\alpha+1, \beta+1)}$.

Now, in view of (2.5), (2.13), and (2.22), the rest of a proof may be completed by mathematical induction.

For a piecewise constant function $f$ with $M$ discontinuities at the points $x_{m}$, $m=1,2, \ldots, M$, Lemma2.2 instantly implies the following three identities $(i \leq M)$ :

$$
\begin{aligned}
& a_{k}^{(i)}\left(x_{1}, x_{2}, \ldots, x_{i}\right)=\sum_{m=i+1}^{M}[f]\left(x_{m}\right) \rho^{(\alpha+1, \beta+1)}\left(x_{m}\right) P_{k}^{(\alpha+1, \beta+1)}\left(x_{m}\right) \prod_{s=1}^{i}\left(x_{s}-x_{m}\right), \\
& a_{k}^{(M-1)}\left(x_{1}, \ldots, x_{m-1}, x_{m+1}, \ldots, x_{M}\right) \\
& \quad=[f]\left(x_{m}\right) \rho^{(\alpha+1, \beta+1)}\left(x_{m}\right) P_{k}^{(\alpha+1, \beta+1)}\left(x_{m}\right) \prod_{s=1 ; s \neq m}^{M}\left(x_{s}-x_{m}\right),
\end{aligned}
$$

and

$$
a_{k}^{(i)}=(-1)^{i} \sum_{m=1}^{M}[f]\left(x_{m}\right) \rho^{(\alpha+1, \beta+1)}\left(x_{m}\right) P_{k}^{(\alpha+1, \beta+1)}\left(x_{m}\right)\left(x_{m}\right)^{i} .
$$

Lemma 2.3. Let $f \in C^{-1}[-1,1]$ and suppose

$$
\begin{aligned}
Q_{M}(x) \equiv Q_{M}\left(t_{1}, \ldots, t_{M}, x\right) & \equiv\left(x-t_{1}\right)\left(x-t_{2}\right) \ldots\left(x-t_{M}\right) \\
& \equiv x^{M}+\sum_{i=1}^{M}(-1)^{i} q_{M-i}^{(M)}\left(t_{1}, t_{2}, \ldots, t_{M}\right) x^{M-i}
\end{aligned}
$$

Then

$$
a_{k}^{(M)}\left(f, t_{1}, t_{2}, \ldots, t_{M}\right)=\sum_{i=0}^{M-1} a_{k}^{(i)}(f) q_{i}^{(M)}\left(t_{1}, t_{2}, \ldots, t_{M}\right)+a_{k}^{(M)}(f)
$$

for $M \in N$ and $k \geq M$.

Proof. First of all, let us mention that the coefficient $q_{i}^{(M)} \equiv q_{i}^{(M)}\left(t_{1}, t_{2}, \ldots, t_{M}\right)$, $i=0,1, \ldots, M-1$, represents the $i$ th elementary symmetric function of the $M$ numbers $t_{1}, t_{2}, \ldots, t_{M}$ (cf. [12, p. 41]); i.e.,

$$
q_{M-i}^{(M)}=\sum_{1 \leq m_{1}<\ldots<m_{i} \leq M} \prod_{j=1}^{i} t_{m_{j}} .
$$


We will prove identity (2.27) by mathematical induction. Let $M=1$. Then $(2.26)$ implies

$$
t_{1}=q_{0}^{(1)} .
$$

From (2.13), on the other hand, it follows that

$$
\begin{aligned}
a_{k}^{(1)}\left(t_{1}\right) & =A_{k} a_{k+1}^{(0)}+\left(t_{1}+B_{k}\right) a_{k}^{(0)}+C_{k} a_{k-1}^{(0)} \\
& =a_{k}^{(0)} t_{1}+A_{k} a_{k+1}^{(0)}+B_{k} a_{k}^{(0)}+C_{k} a_{k-1}^{(0)}=a_{k}^{(0)} t_{1}+a_{k}^{(1)},
\end{aligned}
$$

which combined with (2.29) produces the desired result for $M=1$. (Here and below we use the same abbreviations as in Lemma 2.2 i.e., $A_{k} \equiv A_{k}^{(\alpha+1, \beta+1)}$, etc.)

Now we assume that identity (2.27) is correct for $M=m$ and we will prove it for $M=m+1$. According to our assumption (2.27) and (2.13) we have $(k \geq M)$

$$
\begin{aligned}
a_{k}^{(m+1)}\left(t_{1}, \ldots, t_{m+1}\right)= & A_{k}\left(a_{k+1}^{(0)} q_{0}^{(m)}+\ldots+a_{k+1}^{(m-1)} q_{m-1}^{(m)}+a_{k+1}^{(m)}\right) \\
& +\left(t_{m+1}+B_{k}\right)\left(a_{k}^{(0)} q_{0}^{(m)}+\ldots+a_{k}^{(m-1)} q_{m-1}^{(m)}+a_{k}^{(m)}\right) \\
& +C_{k}\left(a_{k-1}^{(0)} q_{0}^{(m)}+\ldots+a_{k-1}^{(m-1)} q_{m-1}^{(m)}+a_{k-1}^{(m)}\right) \\
= & a_{k}^{(0)} q_{0}^{(m)} t_{m+1} \\
& +\sum_{i=0}^{m-2}\left[\left(A_{k} a_{k+1}^{(i)}+B_{k} a_{k}^{(i)}+C_{k} a_{k-1}^{(i)}\right) q_{i}^{(n)}+a_{k}^{(i+1)} q_{i+1}^{(m)} t_{m+1}\right] \\
& +\left(A_{k} a_{k+1}^{(m-1)}+B_{k} a_{k}^{(m-1)}+C_{k} a_{k-1}^{(m-1)}\right) q_{m-1}^{(m)}+a_{k}^{(m)} t_{m+1} \\
& +A_{k} a_{k+1}^{(m)}+B_{k} a_{k}^{(m)}+C_{k} a_{k-1}^{(m)} \\
= & a_{k}^{(0)} q_{0}^{(m)} t_{m+1}+\sum_{i=0}^{m-2}\left[a_{k}^{(i+1)} q_{i}^{(m)}+a_{k}^{(i+1)} q_{i+1}^{(m)} t_{m+1}\right] \\
& +a_{k}^{(m)} q_{m-1}^{(m)}+a_{k}^{(m)} t_{m+1}+a_{k}^{(m+1)} \\
= & \sum_{i=0}^{m} a_{k}^{(i)} q_{i}^{(m+1)}+a_{k}^{(m+1)},
\end{aligned}
$$

since by $q_{i}^{(m)}+q_{i+1}^{(m)} t_{m+1}=q_{i+1}^{(m+1)}$ for $i=-1,0, \ldots, m-1$, where $q_{-1}^{(m)}=0$ and $q_{m}^{(m)}=1$.

Finally, since solvability of the system of linear equations (2.16) depends on invertibility of the matrix (2.14), let us study the matrix $\mathbf{A}_{M}^{(n)}(f)$ in more details for a function $f \in \tilde{C}^{3}[-1,1]$.

If a function $f \in \tilde{C}^{3}[-1,1]$ has jump discontinuities at $x_{m}, m=1,2, . ., M$, then it can be represented as

$$
f=\sum_{i=0}^{3} \sum_{m=1}^{M}\left[f^{(i)}\right]\left(x_{m}\right) H_{i}\left(x_{m}, \cdot\right)+f_{c} \equiv \sum_{i=0}^{3} H^{(i)}+f_{c} \equiv H^{(0)}+F_{c},
$$

where $f_{c} \in C^{3}[-1,1]$ and $F_{c} \in C[-1,1]$. Thus,

$$
\mathbf{A}_{M}^{(n)}(f)=\mathbf{A}_{M}^{(n)}\left(H^{(0)}\right)+\mathbf{A}_{M}^{(n)}\left(F_{c}\right),
$$


and if

$$
\operatorname{det}\left(\mathbf{A}_{M}^{(n)}\left(H^{(0)}\right)\right) \neq 0
$$

and

$$
\left\|\left(\mathbf{A}_{M}^{(n)}\left(H^{(0)}\right)\right)^{-1}\right\|\left\|\mathbf{A}_{M}^{(n)}\left(F_{c}\right)\right\|<K<1
$$

then by virtue of the Perturbation Lemma [11, p. 74] $\mathbf{A}_{M}^{(n)}(f)$ is also nonsingular and

$$
\left\|\left(\mathbf{A}_{M}^{(n)}(f)\right)^{-1}\right\| \leq \frac{\left\|\left(\mathbf{A}_{M}^{(n)}\left(H^{(0)}\right)\right)^{-1}\right\|}{1-\left\|\left(\mathbf{A}_{M}^{(n)}\left(H^{(0)}\right)\right)^{-1}\right\|\left\|\mathbf{A}_{M}^{(n)}\left(F_{c}\right)\right\|} .
$$

It is known (cf. [11, p. 70]) that

$$
\|\mathbf{A}\|=\max _{1 \leq i \leq M} \sum_{j=1}^{M}\left|a_{i j}\right|
$$

for a matrix $\mathbf{A}=\left(a_{i j}\right)_{i, j=1}^{M}$.

Thus, in view of (2.6), (2.11)-(2.14), (2.32), (2.37), and (3.3),

$$
\left\|\mathbf{A}_{M}^{(n)}\left(F_{c}\right)\right\|=O\left(\frac{1}{n^{3 / 2}}\right)\left[\sum_{m=1}^{M}\left|\left[f^{\prime}\right]\left(x_{m}\right)\right| \rho^{(\alpha / 2+3 / 4, \beta / 2+3 / 4)}\left(x_{m}\right)+o(1)\right] .
$$

Substituting 2.25) into (2.14), it is easy to check that the matrix $\mathbf{A}_{M}^{(n)}\left(H^{(0)}\right)$ may be represented as the product of four matrices

$$
\begin{aligned}
\mathbf{A}_{M}^{(n)}\left(H^{(0)}\right)= & \left((-1)^{i+1} \delta_{i j}\right)_{i, j=1}^{M} \times\left(\left(x_{j+1}\right)^{i}\right)_{i, j=0}^{M-1} \\
& \times\left([f]\left(x_{i}\right) \rho^{(\alpha+1, \beta+1)}\left(x_{i}\right) \delta_{i j}\right)_{i, j=1}^{M} \times \mathbf{P}_{M}^{(n)}\left(x_{1}, \ldots, x_{M}\right) \\
\equiv & \boldsymbol{\Delta} \times \mathbf{V} \times \mathbf{F} \times \mathbf{P}
\end{aligned}
$$

where the second matrix in the product is an $M \times M$ Vandermonde matrix, the third matrix is diagonal, and the forth matrix is defined by (2.17).

Since the first three matrices in this representation are nonsingular, it follows that condition (2.34) is equivalent to the condition $\operatorname{det} \mathbf{P} \neq 0$.

Furthermore, if $\mathbf{P}$ is nonsingular, then by virtue of (2.39) and the inequality $\|\mathbf{A} \times \mathbf{B}\| \leq\|\mathbf{A}\|\|\mathbf{B}\|[11$, p. 70] we have

$$
\|\boldsymbol{\Delta}\|^{-1}|| \mathbf{V}\left\|^{-1}|| \mathbf{F}\right\|^{-1}|| \mathbf{P}^{-1}\|\leq\|\left(\mathbf{A}_{M}^{(n)}\left(H^{(0)}\right)\right)^{-1}\|\leq\| \boldsymbol{\Delta}^{-1}\left|\left\|\left|\mathbf{V}^{-1}\right|\right\|\right| \mathbf{F}^{-1}\left|\left\|\mid \mathbf{P}^{-1}\right\| .\right.
$$

However, it is known [7] that

$$
\left\|\mathbf{V}^{-1}\right\| \leq \max _{1 \leq m \leq M} \prod_{k=1 ; k \neq m}^{M} \frac{1+\left|x_{k}\right|}{\left|x_{m}-x_{k}\right|}
$$


Hence, combining (2.39)-(2.41), we get

$$
\begin{aligned}
& \frac{1}{M} \min _{1 \leq m \leq M} \frac{1}{\left|[f]\left(x_{m}\right)\right| \rho^{(\alpha+1, \beta+1)}\left(x_{m}\right)}\left\|\left(\mathbf{P}_{M}^{(n)}\left(x_{1}, x_{2}, \ldots, x_{M}\right)\right)^{-1}\right\| \\
& \leq\left\|\left(\mathbf{A}_{M}^{(n)}\left(H^{(0)}\right)\right)^{-1}\right\| \\
& \leq \max _{1 \leq m \leq M} \prod_{k=1 ; k \neq m}^{M} \frac{2}{\left|x_{m}-x_{k}\right|} \\
& \times \max _{1 \leq m \leq M} \frac{1}{\left|[f]\left(x_{m}\right)\right| \rho^{(\alpha+1, \beta+1)}\left(x_{m}\right)}\left\|\left(\mathbf{P}_{M}^{(n)}\left(x_{1}, x_{2}, \ldots, x_{M}\right)\right)^{-1}\right\|,
\end{aligned}
$$

where by virtue of (2.8) and (2.17)

$$
\begin{aligned}
O\left(n^{1 / 2}\right) \min _{1 \leq m \leq M} \rho^{(\alpha / 2+3 / 4, \beta / 2+3 / 4)}\left(x_{m}\right) & \leq\left\|\mathbf{P}_{M}^{(n)}\right\|^{-1} \\
& \leq\left\|\left(\mathbf{P}_{M}^{(n)}\right)^{-1}\right\| .
\end{aligned}
$$

Together, (2.43) with (2.38), and (2.42) imply that condition (2.35) of the Perturbation Lemma will be satisfied for sufficiently large $n$.

The importance of the matrix $\mathbf{P}_{M}^{(n)}\left(x_{1}, x_{2}, \ldots, x_{M}\right)$ should be clear now, as nonsingularity of the matrix $\mathbf{A}_{M}^{(n)}(f)$ and the magnitude of its inverse depend on it.

It is easy to construct matrices $\mathbf{P}_{M}^{(k)}\left(x_{1}, x_{2}, \ldots, x_{M}\right)$ such that

$$
\operatorname{det} \mathbf{P}_{M}^{(k)}\left(x_{1}, x_{2}, \ldots, x_{M}\right)=0
$$

for $M$ consecutive indices. Just pick $x_{1}, x_{2}, \ldots, x_{M}$ to be the zeros of $P_{n+M-1}^{(\alpha+1, \beta+1)}(x)$ and consider $\mathbf{P}_{M}^{(k)}\left(x_{1}, x_{2}, \ldots, x_{M}\right)$ for $k=n, n+1, \ldots, n+M-1$.

However,

Lemma 2.4. For any fixed $x_{i} \neq x_{j}, i \neq j$, and $n \in N$, at least one out of $M+1$ consecutive matrices $\mathbf{P}_{M}^{(k)}\left(x_{1}, x_{2}, \ldots, x_{M}\right), k=n, n+1, \ldots, n+M$, is nonsingular.

Proof. $M=1$. This case is trivial. Indeed, by (2.17), $\mathbf{P}_{1}^{(k)}\left(x_{1}\right)=\left(P_{k}\left(x_{1}\right)\right)$. (Here and below we use the same abbreviation as in (2.21) and (2.22).) Thus, invertibility of the matrix depends on whether $x_{1}$ is a zero of the polynomial $P_{k}(x)$. By (2.2) $P_{k}\left(x_{1}\right) \neq 0$ for at least one $k=n, n+1$.

$M=2$. Let us assume that for some $n \in N$, $\operatorname{det} \mathbf{P}_{2}^{(k)}\left(x_{1}, x_{2}\right)=0$ for $k=$ $n, n+1, n+2$. Thus

$$
\begin{gathered}
P_{n}\left(x_{1}\right) P_{n+1}\left(x_{2}\right)-P_{n}\left(x_{2}\right) P_{n+1}\left(x_{1}\right)=0, \\
P_{n+1}\left(x_{1}\right) P_{n+2}\left(x_{2}\right)-P_{n+1}\left(x_{2}\right) P_{n+2}\left(x_{1}\right)=0, \\
P_{n+2}\left(x_{1}\right) P_{n+3}\left(x_{2}\right)-P_{n+2}\left(x_{2}\right) P_{n+3}\left(x_{1}\right)=0 .
\end{gathered}
$$

Since $x_{1} \neq x_{2}$, the combination of (2.1) with (2.44)-(2.46) leads to

$$
P_{n+1}\left(x_{1}\right) P_{n+1}\left(x_{2}\right)=0
$$

and

$$
P_{n+2}\left(x_{1}\right) P_{n+2}\left(x_{2}\right)=0 .
$$

Without loss of generality let us assume that $P_{n+2}\left(x_{1}\right)=0$. Then, by (2.2) it follows that $P_{n+1}\left(x_{1}\right) \neq 0$. Thus (2.47) implies $P_{n+1}\left(x_{2}\right)=0$, which combined with (2.44) implies $P_{n+1}\left(x_{1}\right)=0$, an obvious contradiction. 
$M \geq 3$. The rest of the proof is based on mathematical induction. Let us introduce the additional notation $\mathbf{X}_{m} \equiv\left(\delta_{i j} x_{i}\right)_{i, j=1}^{m}$. Then, by (2.5) and (2.17)

$$
\mathbf{X}_{m} \mathbf{p}_{k}^{(m)}=-\left(A_{k} \mathbf{p}_{k+1}^{(m)}+B_{k} \mathbf{p}_{k}^{(m)}+C_{k} \mathbf{p}_{k-1}^{(m)}\right)
$$

for $k \geq 2$.

Now, assuming that Lemma 2.4 is correct for $M=m-1$, we prove it for the $M=m$ case.

According to our assumption, at least one system out of the systems of vectors $\left\{\mathbf{p}_{k}^{(m)}, \mathbf{p}_{k+1}^{(m)}, \ldots, \mathbf{p}_{k+m-2}^{(m)}\right\}, k=n+1, n+2, \ldots, n+m$, will be linearly independent. Without loss of generality, let as assume that

$$
\operatorname{dim}\left(\operatorname{Span}\left\{\mathbf{p}_{n+1}^{(m)}, \mathbf{p}_{n+2}^{(m)}, \ldots, \mathbf{p}_{n+m-1}^{(m)}\right\}\right)=m-1 .
$$

Now let us assume to the contrary that

$$
\operatorname{dim}\left(\operatorname{Span}\left(\left\{\mathbf{p}_{k}^{(m)}, \mathbf{p}_{k+1}^{(m)}, \ldots, \mathbf{p}_{k+m-1}^{(m)}\right\}\right)\right)<m
$$

for $k=n, n+1, \ldots, n+m$.

Combining (2.49) and (2.51), it is easy to check that the linear operator $T(\mathbf{y})=$ $\mathbf{X}_{m} \mathbf{y}, T: R^{m} \rightarrow R^{m}$, maps the subspace $\operatorname{Span}\left\{\mathbf{p}_{n+1}^{(m)}, \mathbf{p}_{n+2}^{(m)}, \ldots, \mathbf{p}_{n+m-1}^{(m)}\right\}$ into itself.

Since the consecutive Jacobi polynomials do not have common zeros (see (2.2)), there exists a constant $K$ such that the vector $\mathbf{q} \equiv\left(q\left(x_{1}\right), q\left(x_{2}\right), \ldots, q\left(x_{M}\right)\right) \equiv$ $\mathbf{p}_{n+1}^{(m)}+K \mathbf{p}_{n+2}^{(m)}$ has no zero entry, i.e., $q\left(x_{i}\right) \neq 0, i=1,2, \ldots, m$.

Now, repeatedly applying the linear operator $T$ to the vector $\mathbf{q}$, we have $\left(\mathbf{X}_{m}\right)^{s} \mathbf{q}$ $\in \operatorname{Span}\left\{\mathbf{p}_{n+1}^{(m)}, \mathbf{p}_{n+2}^{(m)}, \ldots, \mathbf{p}_{n+m-1}^{(m)}\right\}, s=0,1, \ldots, m-1$, and

$$
\operatorname{det}\left(\mathbf{q}, \mathbf{X}_{m} \mathbf{q}, \ldots,\left(\mathbf{X}_{m}\right)^{m-1} \mathbf{q}\right)=\prod_{i=1}^{m} q\left(x_{i}\right) \operatorname{det} \mathbf{V} \neq 0
$$

where $\mathbf{V}$ is an $m \times m$ Vandermonde matrix with distinct $x_{i} \neq x_{k}, i \neq k$, entries, and that contradicts (2.50).

The magnitude of $\left\|\left(\mathbf{P}_{M}^{(k)}\left(x_{1}, x_{2}, \ldots, x_{M}\right)\right)^{-1}\right\|$ is much harder to analyze. For $M=1$, by virtue of (2.3), the measure of the set $\left\{x \mid x \in[-1,1]\right.$ and $\left\|\left(\mathbf{P}_{1}^{(k)}(x)\right)^{-1}\right\|=$ $\left.1 /\left|P_{n}^{(\alpha, \beta)}(x)\right|>K n^{-1 / 2}\right\}$ is $O(1 / K)$. In general, we do not have estimates for $\left\|\left(\mathbf{P}_{M}^{(k)}\left(x_{1}, x_{2}, \ldots, x_{M}\right)\right)^{-1}\right\|$, although numerical simulations show that typically the norms are not large.

Finally, combining (2.36), (2.38), (2.42), and (2.43), we obtain the following rough estimate

$$
\begin{aligned}
\left\|\left(\mathbf{A}_{M}^{(n)}(f)\right)^{-1}\right\| \leq & O(1) \max _{1 \leq m \leq M} \frac{1}{\left|[f]\left(x_{m}\right)\right| \rho^{(\alpha+1, \beta+1)}\left(x_{m}\right)} \max _{1 \leq m \leq M} \prod_{k=1 ; k \neq m}^{M} \frac{1}{\left|x_{m}-x_{k}\right|} \\
& \times\left\|\left(\mathbf{P}_{M}^{(n)}\right)^{-1}\right\| K_{0}^{-1},
\end{aligned}
$$


where

$$
\begin{aligned}
& K_{0}=1-O\left(\frac{1}{n^{3 / 2}}\right) K_{1} K_{2}, \\
& K_{1}=\sum_{m=1}^{M}\left|\left[f^{\prime}\right]\left(x_{m}\right)\right| \rho^{(\alpha / 2+3 / 4, \beta / 2+3 / 4)}\left(x_{m}\right)+o(1) \\
& K_{2}=\max _{1 \leq m \leq M} \frac{1}{\| f]\left(x_{m}\right) \mid \rho^{(\alpha+1, \beta+1)}\left(x_{m}\right)} \max _{1 \leq m \leq M} \prod_{k=1 ; k \neq m}^{M} \frac{1}{\left|x_{m}-x_{k}\right|}\left\|\left(\mathbf{P}_{M}^{(n)}\right)^{-1}\right\| .
\end{aligned}
$$

\section{MAin Results}

A combination of Lemmas 2.2 and 2.3 leads to the first important result, Theorem 3.1: The locations of discontinuities and the associated jumps of a piecewise constant function with a finite number, $M$, of discontinuities can be recovered exactly in terms of its Fourier-Jacobi coefficients. Indeed, by Lemma 2.2, $a_{k}^{(M)}\left(x_{1}, x_{2}, \ldots, x_{M}\right)=0$. On the other hand (Lemma 2.3) $a_{k}^{(M)}\left(x_{1}, x_{2}, \ldots, x_{M}\right)=$ $\sum_{i=0}^{M-1} a_{k}^{(i)} q_{i}^{(M)}\left(x_{1}, x_{2}, \ldots, x_{M}\right)+a_{k}^{(M)}$. Therefore, solving the system of linear equations (2.16), we can recover the coefficients of the polynomial (2.15), i.e., (2.26), with the roots equal to the locations of discontinuities of the given piecewise constant function $f$.

In a more general case, Theorem 3.2, approximating the locations of discontinuities of a function of the $\tilde{C}^{3}[-1,1]$ class, we will proceed the following way: Although $a_{k}^{(M)}\left(x_{1}, x_{2}, \ldots, x_{M}\right) \neq 0$ for a function which is not piecewise constant, still it will be shown that $a_{k}^{(M)}\left(x_{1}, x_{2}, \ldots, x_{M}\right) \approx 0$ for sufficiently large $k$. Hence, solving the homogeneous system of linear equations (2.16) instead of the nonhomogeneous system $\sum_{i=0}^{M-1} a_{k}^{(i)} q_{i}^{(M)}\left(x_{1}, x_{2}, \ldots, x_{M}\right)+a_{k}^{(M)}=a_{k}^{(M)}\left(x_{1}, x_{2}, \ldots, x_{M}\right) \approx 0$, $k=n, n+1, \ldots, n+M-1$, we will recover the coefficients of the polynomial (2.15) only approximately. It will be shown in Theorem 3.2 that the roots of the polynomial (2.15) are within $O\left(1 / n^{2}\right)$ to the locations of discontinuities of the given function.

Theorem 3.1. Let $f$ be a piecewise constant function defined on the segment $[-1,1]$ with a finite number, $M$, of discontinuities at the points $x_{m}, m=1,2, \ldots, M$. Also suppose that the matrix $\mathbf{A}_{M}^{(n)}(f)$ 2.14 is nonsingular for some $n \geq M$. Then the solutions of the polynomial equation (2.15) represent the discontinuity locations and the solutions of the linear equations (2.18) represent the associated jumps of the function $f$.

Proof. Let us consider the polynomial $Q_{M}$ (2.26) with $t_{m}=x_{m}, m=1,2, \ldots, M$. In order to prove that the polynomials $Q_{M}^{(n)}$ and $Q_{M}$ have identical roots, it suffices to show that they have identical coefficients, i.e., $q_{i}^{(M)}(n)=q_{i}^{(M)}, i=0,1, \ldots, M-1$.

The coefficients $q_{i}^{(M)}(n)$ are determined by the condition (2.16). Since the function $f$ is piecewise constant, it follows that $a_{k}^{(M)}\left(x_{1}, x_{2}, \ldots, x_{M}\right)=0$ for $k \geq M$ (check 2.23) for $i=M)$, which combined with (2.27) with $t_{m}=x_{m}, m=$ $1,2, \ldots, M$, implies that the coefficients $q_{i}^{(M)}$ satisfy the same condition (2.16).

On the other hand, no other choice for $t_{m}, m=1,2, \ldots, M$, except $t_{m}=x_{m}$, will make it possible that $a_{k}^{(M)}\left(t_{1}, t_{2}, \ldots, t_{M}\right)=0$ for $k=n, n+1, \ldots, n+M-1$. Indeed, 
since $\operatorname{det} \mathbf{A}_{M}^{(n)}(f) \neq 0,(2.19)$ implies that $\prod_{s=1}^{M}\left(t_{s}-x_{m}\right)=0, m=1,2, \ldots, M$, which is possible only if $t_{m}=x_{m}$.

Finally, if $x_{m}(n)=x_{m}, m=1,2, \ldots, M$, the linear equations (2.18) and (2.24) are identical. Thus $f_{m}(n)=[f]\left(x_{m}\right), m=1,2, \ldots, M$.

Now we study the accuracy of the approximation to the locations of discontinuities for a function of the $\tilde{C}^{r}[-1,1]$ class.

Theorem 3.2. Suppose the function $f$ belongs to $\tilde{C}^{3}[-1,1], M \geq 2$. In addition, let us assume that the matrix $\mathbf{A}_{M}^{(n)}(f)$ 2.14 is nonsingular for some $n \geq M+3$. Then

$$
\begin{aligned}
x_{m}(n)=x_{m}+O & \left(\frac{1}{n^{5 / 2}}\right) \frac{\left\|\left(\mathbf{A}_{M}^{(n)}\right)^{-1}\right\|}{\prod_{i, j=1 ; i<j}^{M}\left(x_{i}-x_{j}\right)} \\
& \times\left(\sum_{m=1}^{M}\left|\left[f^{\prime}\right]\left(x_{m}\right)\right| \rho^{(\alpha / 2+3 / 4, \beta / 2+3 / 4)}\left(x_{m}\right)+o(1)\right),
\end{aligned}
$$

where $x_{m}(n), m=1,2, \ldots, M$, are the roots of the polynomial equation (2.15).

Proof. The following is an outline of the proof: First we obtain an estimate for the difference between the coefficients of polynomials $Q_{M}^{(n)}$ and $Q_{M}$; then we estimate the difference between the roots of those polynomials.

Since the function $f$ belongs to $\tilde{C}^{3}[-1,1]$, it can be represented as in (2.32).

Due to (2.11) and (2.32),

$$
\begin{aligned}
a_{k}^{(\alpha, \beta)}(f)= & \sum_{i=0}^{3} \sum_{m=1}^{M}\left[f^{(i)}\right]\left(x_{m}\right) \frac{(k-i-1) !}{2^{i+1} k !} \rho^{(\alpha+i+1, \beta+i+1)}\left(x_{m}\right) P_{k-i-1}^{(\alpha+i+1, \beta+i+1)}\left(x_{m}\right) \\
& +a_{k}^{(\alpha, \beta)}\left(f_{c}\right) .
\end{aligned}
$$

Hence,

$$
\begin{aligned}
a_{k}^{(0)}= & 2(k+1) a_{k+1}^{(\alpha, \beta)}(f)=\sum_{m=1}^{M}[f]\left(x_{m}\right) \rho^{(\alpha+1, \beta+1)}\left(x_{m}\right) P_{k}^{(\alpha+1, \beta+1)}\left(x_{m}\right) \\
& +\frac{1}{2 k} \sum_{m=1}^{M}\left[f^{\prime}\right]\left(x_{m}\right) \rho^{(\alpha+2, \beta+2)}\left(x_{m}\right) P_{k-1}^{(\alpha+2, \beta+2)}\left(x_{m}\right) \\
& +\frac{1}{4 k(k-1)} \sum_{m=1}^{M}\left[f^{\prime \prime}\right]\left(x_{m}\right) \rho^{(\alpha+3, \beta+3)}\left(x_{m}\right) P_{k-2}^{(\alpha+3, \beta+3)}\left(x_{m}\right) \\
& +\frac{1}{8 k(k-1)(k-2)} \sum_{m=1}^{M}\left[f^{(3)}\right]\left(x_{m}\right) \rho^{(\alpha+4, \beta+4)}\left(x_{m}\right) P_{k-3}^{(\alpha+4, \beta+4)}\left(x_{m}\right) \\
& +a_{k}^{(0)}\left(f_{c}\right)
\end{aligned}
$$

for $k \geq 3$, where $a_{k}^{(0)}\left(f_{c}\right)=o\left(k^{-5 / 2}\right)$ by virtue of (2.11). 
By (2.5), (2.6), (2.13), (2.23), and (3.3) we have

$$
\begin{aligned}
a_{k}^{(1)}\left(x_{1}\right)= & A_{k}^{(\alpha+1, \beta+1)} a_{k+1}^{(0)}+\left(x_{1}+B_{k}^{(\alpha+1, \beta+1)}\right) a_{k}^{(0)}+C_{k}^{(\alpha+1, \beta+1)} a_{k-1}^{(0)} \\
= & \sum_{m=2}^{M}[f]\left(x_{m}\right) \rho^{(\alpha+1, \beta+1)}\left(x_{m}\right) P_{k}^{(\alpha+1, \beta+1)}\left(x_{m}\right)\left(x_{1}-x_{m}\right) \\
& +\frac{1}{2} \sum_{m=1}^{M}\left[f^{\prime}\right]\left(x_{m}\right) \rho^{(\alpha+2, \beta+2)}\left(x_{m}\right) \\
& \times\left[\frac{A_{k}^{(\alpha+1, \beta+1)}}{k+1} P_{k}^{(\alpha+2, \beta+2)}\left(x_{m}\right)+\frac{x_{1}+B_{k}^{(\alpha+1, \beta+1)}}{k} P_{k-1}^{(\alpha+2, \beta+2)}\left(x_{m}\right)\right. \\
& \left.+\frac{C_{k}^{(\alpha+1, \beta+1)}}{k-1} P_{k-2}^{(\alpha+2, \beta+2)}\left(x_{m}\right)\right] \\
& +\frac{1}{4} \sum_{m=1}^{M}\left[f^{\prime \prime}\right]\left(x_{m}\right) \rho^{(\alpha+3, \beta+3)}\left(x_{m}\right) \\
& \times\left[\frac{A_{k}^{(\alpha+1, \beta+1)}}{(k+1) k} P_{k-1}^{(\alpha+3, \beta+3)}\left(x_{m}\right)+\frac{x_{1}+B_{k}^{(\alpha+1, \beta+1)}}{k(k-1)} P_{k-2}^{(\alpha+3, \beta+3)}\left(x_{m}\right)\right. \\
& \left.+\frac{C_{k}^{(\alpha+1, \beta+1)}}{(k-1)(k-2)} P_{k-3}^{(\alpha+3, \beta+3)}\left(x_{m}\right)\right]+\ldots+a_{k}^{(1)}\left(f_{c}, x_{1}\right) \\
\equiv & I_{1}+I_{2}+I_{3}+I_{4}+o\left(\frac{1}{k^{5 / 2}}\right) .
\end{aligned}
$$

By virtues of (2.7) and since $(k+1)^{-1}=k^{-1}-(k(k+1))^{-1}$ and $(k-1)^{-1}=$ $k^{-1}+(k(k-1))^{-1}$, we have

$$
\begin{aligned}
I_{2}= & \frac{1}{2} \sum_{m=1}^{M}\left[f^{\prime}\right]\left(x_{m}\right) \rho^{(\alpha+2, \beta+2)}\left(x_{m}\right) \\
& \times\left[\left(\frac{1}{k}-\frac{1}{k(k+1)}\right)\left(A_{k-1}^{(\alpha+2, \beta+2)}+O\left(\frac{1}{k^{2}}\right)\right) P_{k}^{(\alpha+2, \beta+2)}\left(x_{m}\right)\right. \\
& +\frac{1}{k}\left(x_{1}+B_{k-1}^{(\alpha+2, \beta+2)}+O\left(\frac{1}{k^{2}}\right)\right) P_{k-1}^{(\alpha+2, \beta+2)}\left(x_{m}\right) \\
& \left.+\left(\frac{1}{k}+\frac{1}{k(k-1)}\right) C_{k-1}^{(\alpha+2, \beta+2)} P_{k-2}^{(\alpha+2, \beta+2)}\left(x_{m}\right)\right] \\
\equiv & I_{2,1}+I_{2,2},
\end{aligned}
$$

where by (2.5),

$$
\begin{aligned}
I_{2,1}= & \frac{1}{2 k} \sum_{m=1}^{M}\left[f^{\prime}\right]\left(x_{m}\right) \rho^{(\alpha+2, \beta+2)}\left(x_{m}\right) \\
& \times\left[A_{k-1}^{(\alpha+2, \beta+2)} P_{k}^{(\alpha+2, \beta+2)}\left(x_{m}\right)+\left(x_{1}+B_{k-1}^{(\alpha+2, \beta+2)}\right) P_{k-1}^{(\alpha+2, \beta+2)}\left(x_{m}\right)\right. \\
& \left.+C_{k-1}^{(\alpha+2, \beta+2)} P_{k-2}^{(\alpha+2, \beta+2)}\left(x_{m}\right)\right] \\
= & \frac{1}{2 k} \sum_{m=2}^{M}\left[f^{\prime}\right]\left(x_{m}\right) \rho^{(\alpha+2, \beta+2)}\left(x_{m}\right) P_{k-1}^{(\alpha+2, \beta+2)}\left(x_{m}\right)\left(x_{1}-x_{m}\right)
\end{aligned}
$$


and by (2.6) and (2.8),

$$
\begin{aligned}
\left|I_{2,2}\right| & =O\left(\frac{1}{k^{2}}\right) \sum_{m=1}^{M}\left|\left[f^{\prime}\right]\left(x_{m}\right)\right| \rho^{(\alpha+2, \beta+2)}\left(x_{m}\right) \sum_{i=k-2}^{k}\left|P_{i}^{(\alpha+2, \beta+2)}\left(x_{m}\right)\right| \\
& =O\left(\frac{1}{k^{5 / 2}}\right) \sum_{m=1}^{M}\left|\left[f^{\prime}\right]\left(x_{m}\right)\right| \rho^{(\alpha / 2+3 / 4, \beta / 2+3 / 4)}\left(x_{m}\right) .
\end{aligned}
$$

Analogously,

$$
\begin{aligned}
I_{3}= & \frac{1}{4 k(k-1)} \sum_{m=2}^{M}\left[f^{\prime \prime}\right]\left(x_{m}\right) \rho^{(\alpha+3, \beta+3)}\left(x_{m}\right) P_{k-2}^{(\alpha+3, \beta+3)}\left(x_{m}\right)\left(x_{1}-x_{m}\right) \\
& +O\left(\frac{1}{k^{7 / 2}}\right) \sum_{m=1}^{M}\left|\left[f^{\prime \prime}\right]\left(x_{m}\right)\right| \rho^{(\alpha / 2+5 / 4, \beta / 2+5 / 4)}\left(x_{m}\right)
\end{aligned}
$$

and $I_{4}=O\left(k^{-9 / 2}\right)$.

Combining (3.4)-(3.8), we obtain

$$
\begin{aligned}
a_{k}^{(1)}\left(x_{1}\right)= & \sum_{m=2}^{M}[f]\left(x_{m}\right) \rho^{(\alpha+1, \beta+1)}\left(x_{m}\right) P_{k}^{(\alpha+1, \beta+1)}\left(x_{m}\right)\left(x_{1}-x_{m}\right) \\
& +\frac{1}{2 k} \sum_{m=2}^{M}\left[f^{\prime}\right]\left(x_{m}\right) \rho^{(\alpha+2, \beta+2)}\left(x_{m}\right) P_{k-1}^{(\alpha+2, \beta+2)}\left(x_{m}\right)\left(x_{1}-x_{m}\right) \\
& +\frac{1}{4 k(k-1)} \sum_{m=2}^{M}\left[f^{\prime \prime}\right]\left(x_{m}\right) \rho^{(\alpha+3, \beta+3)}\left(x_{m}\right) P_{k-2}^{(\alpha+3, \beta+3)}\left(x_{m}\right)\left(x_{1}-x_{m}\right) \\
& +O\left(\frac{1}{k^{5 / 2}}\right)\left(\sum_{m=1}^{M}\left|\left[f^{\prime}\right]\left(x_{m}\right)\right| \rho^{(\alpha / 2+3 / 4, \beta / 2+3 / 4)}\left(x_{m}\right)+o(1)\right) .
\end{aligned}
$$

Next, we construct the sequence $a_{k}^{(2)}\left(x_{1}, x_{2}\right), \ldots, a_{k}^{(M)}\left(x_{1}, x_{2}, \ldots, x_{M}\right)$ by the recursion formula (2.13). Subsequently, by (2.6), (2.13), (2.23), and (3.9) we obtain

$$
a_{k}^{(M)}\left(x_{1}, x_{2}, \ldots, x_{M}\right)=O\left(\frac{1}{k^{5 / 2}}\right)\left(\sum_{m=1}^{M}\left|\left[f^{\prime}\right]\left(x_{m}\right)\right| \rho^{(\alpha / 2+3 / 4, \beta / 2+3 / 4)}\left(x_{m}\right)+o(1)\right) .
$$

Furthermore, combining (2.27) with $t_{m}=x_{m}, m=1,2, \ldots, M$, and (3.10), we conclude that the coefficients $q_{i}^{(M)}, i=0,1, \ldots, M-1$, of the algebraic equation (2.26) satisfy the system of linear equations

$$
\sum_{i=0}^{M-1} a_{n+j}^{(i)} q_{i}^{(M)}+a_{n+j}^{(M)}=O\left(\frac{1}{n^{5 / 2}}\right)\left(\sum_{m=1}^{M}\left|\left[f^{\prime}\right]\left(x_{m}\right)\right| \rho^{(\alpha / 2+3 / 4, \beta / 2+3 / 4)}\left(x_{m}\right)+o(1)\right)
$$

for $j=0,1, \ldots, M-1$. Hence, by virtue of (2.16),

$$
\mathbf{q}^{(M)}(n)-\mathbf{q}^{(M)}=\left(\mathbf{A}_{M}^{(n)}\right)^{-1} \mathbf{r}(n),
$$


where $\mathbf{q}^{(M)}(n) \equiv\left(q_{0}^{(M)}(n), \ldots, q_{M-1}^{(M)}(n)\right), \mathbf{q}^{(M)} \equiv\left(q_{0}^{(M)}, \ldots, q_{M-1}^{(M)}\right)$, and

$$
\|\mathbf{r}(n)\|=O\left(\frac{1}{n^{5 / 2}}\right)\left(\sum_{m=1}^{M}\left|\left[f^{\prime}\right]\left(x_{m}\right)\right| \rho^{(\alpha / 2+3 / 4, \beta / 2+3 / 4)}\left(x_{m}\right)+o(1)\right)
$$

is the residual vector.

Thus,

$$
\left\|\mathbf{q}^{(M)}(n)-\mathbf{q}^{(M)}\right\| \leq\left\|\left(\mathbf{A}_{M}^{(n)}\right)^{-1}\right\|\|\mathbf{r}(n)\| .
$$

Now, let us consider the function $F\left(x_{1}, x_{2}, \ldots, x_{M}\right)=\left(q_{M-1}^{(M)}, q_{M-2}^{(M)}, \ldots, q_{0}^{(M)}\right) \in$ $R^{M}$ with the domain $\left\{\left(x_{1}, x_{2}, \ldots, x_{M}\right) \mid-1<x_{1}<x_{2}<\ldots<x_{M}<1\right\}$, i.e., the function mapping the real distinct roots of a monic polynomial on its coefficients (see $(2.26)$ ). Obviously the function $F$ is differentiable and it is not difficult to check that (see (2.28) and [17, Lemma, p. 137])

$$
\operatorname{det}\left(F^{\prime}\left(x_{1}, x_{2}, \ldots, x_{M}\right)\right)=\prod_{i, j=1 ; i<j}^{M}\left(x_{i}-x_{j}\right) \neq 0 .
$$

Hence, the inverse of the function $F$ exists and is differentiable (cf. 21, Theorem 2-11, p. 35]). Later this implies that $F^{-1}$ belongs to the Lip1 class (cf. [21, Lemma $2-10$, p. 35]), i.e.,

$$
\begin{aligned}
\|\mathbf{x}-\mathbf{x}(n)\| & \leq K(M)\left\|\left(F^{-1}\right)^{\prime}\left(F^{-1}\left(x_{1}, \ldots, x_{M}\right)\right)\right\|\left\|\mathbf{q}^{(M)}-\mathbf{q}^{(M)}(n)\right\| \\
& =K(M)\left\|\left(F^{\prime}\left(x_{1}, x_{2}, \ldots, x_{M}\right)\right)^{-1} \mid\right\|\left\|\mathbf{q}^{(M)}-\mathbf{q}^{(M)}(n)\right\|,
\end{aligned}
$$

where $\mathbf{x} \equiv\left(x_{1}, x_{2}, \ldots, x_{M}\right)$ and $\mathbf{x}(n) \equiv\left(x_{1}(n), x_{2}(n), \ldots, x_{M}(n)\right)$. However,

$$
\left(F^{\prime}\left(x_{1}, x_{2}, \ldots, x_{M}\right)\right)^{-1}=\frac{\operatorname{adj}\left(F^{\prime}\left(x_{1}, x_{2}, \ldots, x_{M}\right)\right)}{\operatorname{det}\left(F^{\prime}\left(x_{1}, x_{2}, \ldots, x_{M}\right)\right)}
$$

(cf. [17, p. 334]) and

$$
\left\|\operatorname{adj}\left(F^{\prime}\left(x_{1}, x_{2}, \ldots, x_{M}\right)\right)\right\| \leq K(M)
$$

since $\left|x_{m}\right|<1, m=1,2, \ldots, M$. Thus,

$$
\|\mathbf{x}-\mathbf{x}(n)\| \leq \frac{K(M)}{\left|\operatorname{det}\left(F^{\prime}\left(x_{1}, x_{2}, \ldots, x_{M}\right)\right)\right|}\left\|\mathbf{q}^{(M)}-\mathbf{q}^{(M)}(n)\right\| .
$$

Together (3.13)-(3.15) and (3.17) lead to the estimate (3.1).

The following is an estimate of the accuracy of approximation to jumps.

Theorem 3.3. Suppose the function $f$ belongs to $\tilde{C}^{3}[-1,1], M \geq 2$. In addition, let us assume that $\operatorname{det}\left(\mathbf{A}_{M}^{(n)}(f)\right) \neq 0$ (2.14) and $P_{k}^{(\alpha+1, \beta+1)}\left(x_{m}(n)\right) \neq 0$ for some $n \geq M+3$ and $k \sim n$. Then

$$
\begin{aligned}
& f_{m}(n)=[f]\left(x_{m}\right)+\frac{\|\mathbf{x}(n)-\mathbf{x}\| n^{-1 / 2}}{\rho^{(\alpha+1, \beta+1)}\left(x_{m}(n)\right) P_{k}^{(\alpha+1, \beta+1)}\left(x_{m}(n)\right) \prod_{s=1 ; s \neq m}^{M}\left(x_{s}(n)-x_{m}(n)\right)} \\
& \times\left\{O(n)[f]\left(x_{m}\right) \rho^{(\alpha / 2-1 / 4, \beta / 2-1 / 4)}\left(\xi_{m}(n)\right) \prod_{s=1 ; s \neq m}^{M}\left(x_{s}(n)-x_{m}(n)\right)\right. \\
& \left.+[f]\left(x_{m}\right) \rho^{(\alpha / 2-1 / 4, \beta / 2-1 / 4)}\left(x_{m}\right)+O(1)\right\}
\end{aligned}
$$


for some $\xi_{m}(n) \in\left(\min \left(x_{m}, x_{m}(n)\right), \max \left(x_{m}, x_{m}(n)\right)\right)$.

The proof of Theorem 3.3 is essentially analogous to the proof of Theorem 3.2 and we omit the details.

Estimates for a function with a single jump discontinuity are the same except for the term $\prod_{i, j=1 ; i<j}^{M}\left(x_{i}-x_{j}\right)$.

\section{Description of the ALgorithm AND NUmerical EXAmples}

First, let us clarify the order of accuracy for the estimates (3.1) and (3.18). According to (2.40) and (2.43), roughly the error terms in the estimates (3.1) and (3.18) for a function $f \in \tilde{C}^{3}[-1,1]$ cannot be better than $x_{m}(f, n)-x_{m}(f)=$ $O\left(1 / n^{2}\right)$ and $f_{m}(n)-[f]\left(x_{m}\right)=O(n)\|\mathbf{x}(n)-\mathbf{x}\|=O(1 / n)$, respectively.

Obviously the following question should be addressed: How do we extract information about the exact number, $M$, of discontinuities from a finite number of Fourier-Jacobi coefficients?

We suggest two possible ways to recover the number of discontinuities: First, following Eckhoff [5, p. 688], we pick a trial number $\tilde{M}$, large enough to guarantee that $\tilde{M}>M$. Then the rank of the matrix $\mathbf{A}_{\tilde{M}}^{(n)}$ will equal $M$. Second, we may utilize the identity determining the jumps of a bounded not-too-highly oscillating function by means of its differentiated Fourier-Jacobi partial sums (for more general kernels, see [10]).

Theorem 4.1 ([13]). Let $r \in Z_{+}$and suppose $\Lambda B V$ is the class of functions of $\Lambda$-bounded variation determined by the sequence $\Lambda=\left(\lambda_{k}\right)_{k=1}^{\infty}$. Then the identity

$$
\lim _{n \rightarrow \infty} \frac{\left(S_{n}^{(\alpha, \beta)}(f, x)\right)^{(2 r+1)}}{n^{2 r+1}}=\frac{(-1)^{r}\left(1-x^{2}\right)^{-r-1 / 2}}{(2 r+1) \pi}[f](x)
$$

is valid for every $f \in \Lambda B V$ and each fixed $x \in(-1,1)$, if condition $\Lambda B V \subset H B V$ holds.

$(\Lambda B V$, and in particular $H B V$, is a class of functions with generalized bounded variation. For the exact definition consult 23 .)

According to identity (4.1), for a fixed $r$ and sufficiently large $n$, the function $\left(1-x^{2}\right)^{r+1 / 2}\left|\left(S_{n}^{(\alpha, \beta)}(f, x)\right)^{(2 r+1)}\right| / n^{2 r+1}$ must attain the largest local maximum in the vicinity of the actual points of discontinuity of the function $f$. Hence, we may assume that the number of discontinuities, $M$, equals the number of sharp local spikes of the graph of the differentiated Fourier-Jacobi partial sum.

Our technique might also be usable in conjunction with the other author's methods. For instance, the nonlinear enhancement procedure developed by Gelb and Tadmor in [10] can be used to recover the number of discontinuities, $M$, with more success. Once the nonlinear enhancement is applied, it gives a picture of sharp spikes at the vicinity of the actual locations of discontinuities of a function, removing other oscillatory behavior of the graph (see [10, Figures 5-8, pp. 1406-1407]). Thus, it makes it easier to identify the exact number of discontinuities of a function.

Regarding the norm of $\left(\mathbf{A}_{M}^{(n)}\right)^{-1}$, by virtue of (2.53) , it essentially depends on the behavior of the matrix $\left(\mathbf{P}_{M}^{(n)}\left(x_{1}, x_{2}, \ldots, x_{M}\right)\right)^{-1}$. We suggest using QR factorization to estimate the norm of the matrix $\left(\mathbf{A}_{M}^{(n)}\right)^{-1}$ in order to avoid a sharp decline in the accuracy of approximations. (See [11, p. 75].) 
Let us illustrate a direct application of the method to the following function with two jump discontinuities:

$$
f_{1}(x)= \begin{cases}0 & \text { if }-1<x<-2 / 3 \\ \sqrt{x+1} & \text { if }-2 / 3<x<1 / 2 \\ 0 & \text { if } 1 / 2<x<1\end{cases}
$$

(We are assuming that a finite number of its Fourier-Legendre coefficients are known.)

Utilizing Fourier-Legendre coefficients of the function $f_{1}$, we calculate its higher order Fourier coefficients via the formulas (2.12) and (2.13).

Next, we pick $\tilde{M}=6$ and apply QR factorization to the matrix (2.14) in order to identify the rank of the matrix, i.e., the number of discontinuities of the function $f_{1}\left((-k) \equiv 10^{-k}, k \in Z_{+}\right)$.

The following is the triangular matrix of QR factorization of the matrix (2.14) for $\tilde{M}=6$ :

$$
\left(\begin{array}{cccccc}
-0.55 & 0.19 & -0.15 & 0.04 & -0.04 & 0.005 \\
0 & 0.23 & 0.04 & 0.082 & 0.03 & 0.03 \\
0 & 0 & -1.5(-5) & -9.6(-6) & -1.8(-5) & -1.4(-5) \\
0 & 0 & 0 & -1.2(-5) & -1.1(-5) & -1.4(-5) \\
0 & 0 & 0 & 0 & 7.2(-6) & 6.7(-7) \\
0 & 0 & 0 & 0 & 0 & 4.9(-6)
\end{array}\right) .
$$

It is reasonable to assume that $M=2$.

Next, we calculate the norms of the matrix $\left(\mathbf{A}_{M}^{(n)}\right)^{-1}$ to avoid a sharp decline in the accuracy of approximations. The results of calculation are summarized in Table 1.

TABLE 1. The norms of the matrix $\left(\mathbf{A}_{M}^{(n)}\right)^{-1}$ for various values of $n$.

\begin{tabular}{|c|c|c|c|}
\hline$n$ & 32 & 64 & 128 \\
\hline$\left\|\left(\mathbf{A}_{M}^{(n)}\right)^{-1}\right\|$ & 9.3 & 10.0 & 26.3 \\
\hline
\end{tabular}

Now, the system of linear equations (2.16) is solved and the results are given in Table 2.

Finally, the polynomial equation (2.15) is solved with the coefficients presented in Table 2. The final results are presented in Table 3.

We have tested the theoretical result of Theorem 3.1 via a symbolic computation using Mathematica. The following is a piecewise constant function with ten discontinuities, some of them clustered within 0.0001 distance, and with the corresponding jumps ranging from 0.01 to 100 :

$$
\begin{aligned}
f_{2}(x)= & \frac{1}{2} H_{0}\left(-\frac{999}{1000}, x\right)+\frac{1}{5} H_{0}\left(-\frac{99}{100}, x\right)+\frac{1}{10} H_{0}\left(-\frac{2}{10000}, x\right) \\
& +\frac{1}{100} H_{0}\left(-\frac{1}{10000}, x\right)+H_{0}\left(\frac{1}{1000}, x\right)-\frac{1}{100} H_{0}\left(\frac{2}{1000}, x\right)+H_{0}\left(\frac{1}{2}, x\right) \\
& +10 H_{0}\left(\frac{2}{3}, x\right)+100 H_{0}\left(\frac{998}{1000}, x\right)+H_{0}\left(\frac{999}{1000}, x\right) .
\end{aligned}
$$


TABLE 2. The solutions of the system of linear equations (2.16) for various values of $n$ with absolute errors in the estimates to the coefficients $q_{0}=-1 / 3$ and $q_{1}=-1 / 6$ of the polynomial (2.15).

\begin{tabular}{|c|c|c|c|}
\hline$n$ & 32 & 64 & 128 \\
\hline$q_{0}(n)$ & -0.33354 & -0.333392 & -0.333348 \\
\hline$\left|q_{0}(n)-q_{0}\right|$ & $2.06(-4)$ & $5.86(-5)$ & $1.46(-5)$ \\
\hline$q_{1}(n)$ & -0.167629 & -0.166917 & -0.166731 \\
\hline$\left|q_{1}(n)-q_{1}\right|$ & $9.62(-4)$ & $2.50(-4)$ & $6.43(-5)$ \\
\hline
\end{tabular}

TABLE 3. The solutions of the polynomial equation (2.15) for various values of $n$ with absolute errors in the estimates to the discontinuity locations $x_{1}=-2 / 3$ and $x_{2}=1 / 2$ for function 4.2.

\begin{tabular}{|c|c|c|c|}
\hline$n$ & 32 & 64 & 128 \\
\hline$x_{1}(n)$ & -0.667393 & -0.66686 & -0.666716 \\
\hline$\left|x_{1}-x_{1}(n)\right|$ & $7.26(-4)$ & $1.93(-4)$ & $4.91(-5)$ \\
\hline$x_{2}(n)$ & 0.499765 & 0.499942 & 0.499985 \\
\hline$\left|x_{2}-x_{2}(n)\right|$ & $2.35(-4)$ & $5.76(-5)$ & $1.49(-5)$ \\
\hline
\end{tabular}

All discontinuity locations, as well as the associated jumps, of the function $f_{2}$ have been recovered exactly using its Fourier-Legendre coefficients. We have also considered other piecewise constant functions utilizing its Fourier-Jacobi coefficients with various indices $\alpha>-1$ and $\beta>-1$. For all of them the discontinuity locations have been recovered exactly.

Next, we have considered the function $f_{2}$ perturbed by a smooth function on the interval $[-1,1]$, i.e., $f_{3}(x)=f_{2}(x)+5 /\left(2 x^{2}+x-6\right)$.

Despite the highly clustered discontinuity locations, as well as a large ratio of the magnitudes of the jumps, we found the absolute value of the largest error for approximation to the points of discontinuity and the associated jumps as it is given in Table 4

The following is a function with three jump discontinuities:

$$
f_{4}(x)= \begin{cases}0 & \text { if }-1<x<-1 / 4 \\ e^{x} & \text { if }-1 / 4<x<1 / 3 \\ \sin x & \text { if } 1 / 3<x<2 / 3 \\ 0 & \text { if } 2 / 3<x<1\end{cases}
$$

Below we present the absolute values of the largest error in the estimation of the points of discontinuity and the associated jumps of the function (4.4) obtained by applying the suggested method and summarized in Table 5 . 
TABLE 4. Largest errors in the approximation to the locations and associated jumps of function $f_{3}$ using its Fourier-Legendre coefficients.

\begin{tabular}{|c|c|c|c|}
\hline$n$ & 32 & 64 & 128 \\
\hline Location-error & $4.9(-1)$ & $6.3(-8)$ & $3.8(-35)$ \\
\hline Jump-error & $5.5(-1)$ & $1.1(-5)$ & $6.6(-33)$ \\
\hline
\end{tabular}

TABLE 5. Largest errors in the estimates to the discontinuity locations and the associated jumps for the function (4.4) using its Fourier-Legendre coefficients.

\begin{tabular}{|c|c|c|c|c|}
\hline$n$ & 32 & 64 & 128 & 256 \\
\hline Location-error & $1.2(-3)$ & $2.1(-4)$ & $5.4(-5)$ & $1.3(-5)$ \\
\hline Jump-error & $1.4(-2)$ & $4.0(-3)$ & $2.4(-3)$ & $3.4(-4)$ \\
\hline
\end{tabular}

This is a piecewise polynomial function with five jump discontinuities:

$$
f_{5}(x)= \begin{cases}x^{2}+2 x / 3+10 / 9 & \text { if }-1<x<-1 / 3 \\ x / 6+7 / 144 & \text { if }-1 / 3<x<-1 / 4, \\ x^{2}-1 & \text { if }-1 / 4<x<0 \\ 4 x^{2}-4 x+1 & \text { if } 0<x<1 / 2 \\ 9 x^{2}-12 x+5 & \text { if } 1 / 2<x<2 / 3 .\end{cases}
$$

The absolute value of the largest absolute error in the computed singularity locations and the associated jumps for the function (4.5) are given in Table 6.

TABLE 6. Largest errors in the approximations to the locations and the associated jumps of the function (4.5) using FourierLegendre coefficients.

\begin{tabular}{|c|c|c|c|c|}
\hline$n$ & 32 & 64 & 128 & 256 \\
\hline Location-error & $2.1(-2)$ & $5.0(-4)$ & $1.2(-4)$ & $3.9(-5)$ \\
\hline Jump-error & $6.5(-1)$ & $2.4(-2)$ & $1.1(-2)$ & $6.6(-3)$ \\
\hline
\end{tabular}

\section{ACKNOWLEDGMENTS}

The author thanks the reviewers for some very helpful suggestions. 


\section{REFERENCES}

[1] N. S. Banerjee and J. F. Geer, Exponentially accurate approximations to periodic Lipschitz functions based on Fourier series partial sums, J. Sci. Comput. 13 (1998), 419-460. MR 2000b:65020

[2] R. B. Bauer, "Numerical Shock Capturing Techniques," Doctor. Thesis, Division of Applied Mathematics, Brown University, 1995.

[3] W. Cai, D. Gottlieb, and C.-W. Shu, Essentially nonoscillatory spectral Fourier methods for shock wave calculations, Math. Comp. 52 (1989), 389-410. MR 90a:65212

[4] K. S. Eckhoff, Accurate and efficient reconstruction of discontinuous functions from truncated series expansions, Math. Comp. 61 (1993), 745-763. MR 94a:65073

[5] K. S. Eckhoff, Accurate reconstructions of functions of finite regularity from truncated Fourier series expansions, Math. Comp. 64 (1995), 671-690. MR 95f:65234

[6] K. S. Eckhoff, On a high order numerical method for functions with singularities, Math. Comp. 67 (1998), 1063-1087. MR 98j:65014

[7] W. Gautschi, Norm estimates for inverses of Vandermonde matrices, Numer. Math. 23 (1975), 337-347. MR 51:14550.

[8] J. Geer and N. S. Banerjee, Exponentially accurate approximations to piece-wise smooth periodic functions, J. Sci. Comput. 12 (1997), 253-287. MR 98m:41032

[9] A. Gelb and E. Tadmor, Detection of edges in spectral data, Appl. Comput. Harmon. Anal. 7 (1999) 101-135. MR 2000g:42003

[10] A. Gelb and E. Tadmor, Detection of edges in spectral data II. Nonlinear enhancement, SIAM J. Numer. Anal. 38 (2000), 1389-1408. MR 2001i:42003

[11] G. Hämmerlin and K. Hoffman, "Numerical Analysis," Undergraduate Texts in Mathematics, Springer-Verlag, New York, 1991. MR 92d:65001

[12] R. A. Horn and C. R. Johnson, "Matrix Analysis," Cambridge University Press, Cambridge, 1990. MR 91i:15001

[13] G. Kvernadze, Determination of the jumps of a bounded function by its Fourier series, J. of Approx. Theory 92 (1998), 167-190. MR 99m:42005

[14] G. Kvernadze, T. Hagstrom, and H. Shapiro, Locating discontinuities of a bounded function by the partial sums of its Fourier series, J. Sci. Comput. 4 (1999), 301-327.

[15] G. Kvernadze, T. Hagstrom, and H. Shapiro, Detecting the singularities of a function of $V p$ class by its integrated Fourier series, Comput. Math. Appl. 39 (2000), 25-43. MR 2000m:42005

[16] G. Kvernadze, Approximation of the singularities of a bounded function by the partial sums of its differentiated Fourier series, Appl. Comput. Harmon. Anal. 11 (2001), 439-454. MR 2002i:42001

[17] S. Lang, "Algebra," Addison-Wesley Publishing Company, Inc., Reading, Massachusetts, 1965. MR 33:5416

[18] H. N. Mhaskar and J. Prestin, On a build-up polynomial frame for the detection of singularities, in "Self-Similar Systems"(V. B. Priezzhev and V. P. Spiridonov, Eds.), Joint Institute for Nuclear Research, Dubna, Russia, 1999, pp. 98-109. MR 2003b:94009

[19] H. N. Mhaskar and J. Prestin, Polynomial frames for the detection of singularities, in "Wavelet Analysis and Multiresolution Methods" (Tian-Xiao He, Ed.), Lecture Notes in Pure and Applied Mathematics, Vol. 212, Marcel Decker, 2000, pp. 273-298. MR 2001k:65212

[20] H. N. Mhaskar and J. Prestin, On the detection of singularities of a periodic function, Adv. Comput. Math. 12 (2000), 95-131.

[21] M. Spivak, "Calculus on Manifolds," W. A. Benjamin, Inc., New York, 1965. MR 2001a: 42003

[22] G. Szegő, "Orthogonal Polynomials," Amer. Math. Soc. Colloq. Publ., Vol. 23, Amer. Math. Soc., Providence, RI, 1967. MR 46:9631

[23] D. Waterman, On convergence of Fourier series of functions of generalized bounded variation, Studia Math. 44 (1972), 107-117. MR 46:9623

Department of Mathematics, Weber State University, Ogden, Utah 84408

E-mail address: gkvernadze@weber.edu 